\title{
Design and Implementation of Tanks War Game Based on the Android
}

\section{Platform}

\author{
Biqing $\mathrm{Li}^{1, \text { a }}$,Wenya Lai ${ }^{* 1,}$ a, Chongjun Yang ${ }^{1, a}$, Shiyong Zheng ${ }^{2, b}$ \\ ${ }^{1}$ College of Mechanical and Electronic Engineering, Hezhou university, Hezhou Guangxi \\ 542899, China; \\ ${ }^{2}$ College of Computer Science and Information Engineering, Hezhou university, Hezhou \\ Guangxi 542899, China; \\ ajanliful@163.com, ${ }^{b} 229292710 @ q q . c o m$
}

Keywords:Android Platform,Tank War,Thread.

\begin{abstract}
This paper mainly introduces the design ideas and realization process of the game.The design draw the background of all the objects at the begin of the game.In the thread when the main program was running, the frame refresh will be at certain frequency with double buffering technology to screen redraw and reflect the state of the entire game in real-time. Tanks controlled by users run in the main thread, move along with the refresh frequency of the screen. Tanks will be initialized when the game starts and then drawn on top of the map. The design mainly allows tanks to move and shot shells intelligently, destroy the function of the others,and meets the needs of users.
\end{abstract}

\section{Introduction}

Compared with other application software, the game in Android platform has strong infectiousness; one game player can drive many players surrounding to attend it. As described above, the paper shows the basic flow and core technology of Android platform development by taking Battle City based on Android as the pointcut.

\section{Analysis and Design}

Demand analysis on system function

(1) Start game, select the option of starting game in the selecting menu in the user's angle, press down the confirm key; the computer responses to trigger the pictures of beginning the game for several seconds; then, it begins to trigger the background pictures and tank to complete the game initialization.

(2) display the menu window; the player carries out selection.

(3) select game level; the user confirms it through selecting the level in the menu.

(4) control the tank's direction; when the user emits the direction command to the tank, the computer responses and triggers the algorithm in run() method to carry out plus/minus calculation to the tank's current coordinate after calculation, the tank's current coordinate value is changed and shown on the screen through the tank image of the threading so as to complete the control to the tank moving; and the user directly control the use case.

(5) The bullets emitted by both parties need to be controlled and tracked; it requires the shooting place to be in the independent threading. The player's tank needs to scan the enemy's all tanks in each process; and the enemy's tank only needs to scan the player's tank. 
(6) Shoot bullet; when the user clicks to enter the game, the computer triggers the threading to remap the tank picture on the phone screen. The use case is controlled by the computer directly and the user can't control it.

(7) Control the enemy's tank; the user can't control it; it is controlled by the system.

(8) Both parties need to consider whether their tanks will hit the enemy's tank in the marching process to avoid overlaying in operation and causing many situations inconsistent with the design.

(9) When the user is operating the game, he/she needs the score recording function. It adopts reasonable scoring standard and designs the scoring standard.

(10) The player's camp has a base camp; when the bullet hits the base camp, the computer can judge to terminate the game and control it based on the condition.

(11) Terminate the game; the user in the game can't terminate the game directly; when the life value of tank controlled by the user is zero, the computer can judge to terminate the game and control it based on the condition.

\section{Sysem Realization}

\subsection{Game Login Page Module}

Realize login through verifying the user password input by the user.

3.2 Game Welcome Module

The module realizes the game welcome module; the player can enter the game through clicking the screen.

\subsection{Game Selection Module}

The module completes the realization of function for the player to select tank and enter the game by clicking the tank selected.

A part of codes are shown below:

public MenuView(Context context) \{

super(context);

this.mainActivity = (MainActivity) context;

caidanbj = BitmapFactory.decodeResource(this.getResources(), R.drawable.caidanbj);

tk56 = BitmapFactory.decodeResource(this.getResources(), R.drawable.tk56);

bgSLTank = tk56;

tk53 = BitmapFactory.decodeResource(this.getResources(), R.drawable.tk53);

tk50 = BitmapFactory.decodeResource(this.getResources(), R.drawable.tk50);

tk46 = BitmapFactory.decodeResource(this.getResources(), R.drawable.tk46);

bgS = BitmapFactory.decodeResource(this.getResources(), R.drawable.tk105);

bgSL = BitmapFactory.decodeResource(this.getResources(), R.drawable.tk74);

tmKL = BitmapFactory.decodeResource(this.getResources(), R.drawable.tk40);

tmKR = BitmapFactory.decodeResource(this.getResources(), R.drawable.tk43);

daihao $=$ BitmapFactory.decodeResource(this.getResources(), R.drawable.tk69);

sudu = BitmapFactory.decodeResource(this.getResources(), R.drawable.tk73);

xueliang = BitmapFactory.decodeResource(this.getResources(), R.drawable.tk80);

$\mathrm{zb}=$ BitmapFactory.decodeResource(this.getResources(), R.drawable.tk82);

gege $=$ BitmapFactory.decodeResource(this.getResources(), R.drawable.tk78);

paint = new Paint();

soundPool=new SoundPool(2, AudioManager.STREAM_MUSIC, 100);

sound $=$ soundPool.load(context, R.raw.tk12, 0); 
this.setFocusable(true);

\}

3.4 Realization of game module

The module realizes control to various threadings in the game; through controlling the player's tank, the enemy's tank and the flyer in air, it realizes the game playing. In the game, it inspects the blood amount of the player's tank; if the blood is consumed up before the game over, the game is terminated to be over; if completing the game, it shows the success in breaking through the game.

3.5 Realization of module of succeeding in breaking through the game

This module realizes the display of function of succeeding in breaking through the game

3.6 Realization of module of failing in breaking through the game.

This module realizes the judgment and processing of failing in breaking through the game.

The game interface is shown in Graph 1 as follow:

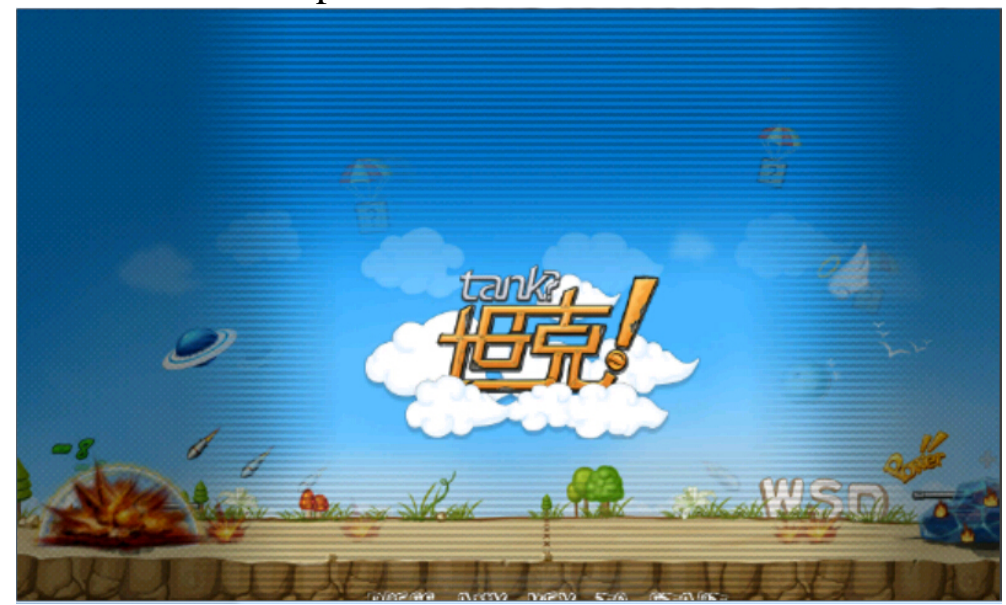

Graph 1 The game interface

\section{Conclusion}

The paper designs and realizes basic functions of Battle City Game; In the aspect of function, the system doesn't provide the function of pausing and beginning the game in the playing process and hasn't realized the function of filing games.

This work is supported the following fund :

2016 The project of improving the basic ability of young teachers in Colleges and universities in Guangxi:"Design and development of electronic commerce platform of agricultural products based on Semantic Technology”(No,KY2016YB455).

2016 Guangxi higher education teaching reform project: "Exploration on the cultivation mechanism of the teaching characteristics of Communication Engineering Specialty Based on ZTE ICT education platform”

2015 college students' innovative training program: "Research on the application of value added travel experience in the mobile terminal of the 'ethnic custom travel' in Guangxi” (No 201511838070);\&\& “The design and development HeYuanTong Campus Mobile Phone APP based on Android" (No 201511838034).

2015 Teaching case project construction project of hezhou university: “Tourist positioning and guiding system project of Huangyao scenic”;\&\& “Development and application of the information management system of 'Huang Yao Tong'”.

Nanling corridor of ethnic culture research base of Open Fund Project: "Nanling National corridor costumes digital display design” (No 2015kf27); 
Project of scientific research and technology development project of Hezhou: "Design and implementation of agricultural products e-commerce platform based on Semantic Technology" (No,Hekeneng 1506006)

Scientific research project of hezhou university: "The intelligent home system Implementation Research based on the Internet of Things technology" (No 2014YBZK10); "Research and development of E-government platform based on mobile terminal” (No 2015ZZZK03);

Reform in Education project of hezhou university: "Internet of things"(No hzxytszy201501); "Research on application personnel training model of Communications for SMEs"(No hzxyjg201525);

Master degree discipline construction scientific research and Cultivation Project: "Research on the social work service of the teenagers' Network Addiction"(No 2015SHGZ005) \&\&"Research on the intelligent development of rural tourism in Guangxi based on the Internet of things" (No 2015MTA16);

\section{References}

[1]. Zhu Guiying. Android Development Application from Introduction to Master[M]. Beijing: Chinese Railway Press, 2010.

[2]. Computernik. Android System Optimization Guidance [J].2011.

[3]. Li Jia. Mobile Internet Service Research Based on Smart Terminal [D]. Central South University, 2009.

[4]. Gai Suolin, Wang Shijiang. Google Android Development Guidance [M]. Beijing: Posts and Telecom Press . 2011.11. 